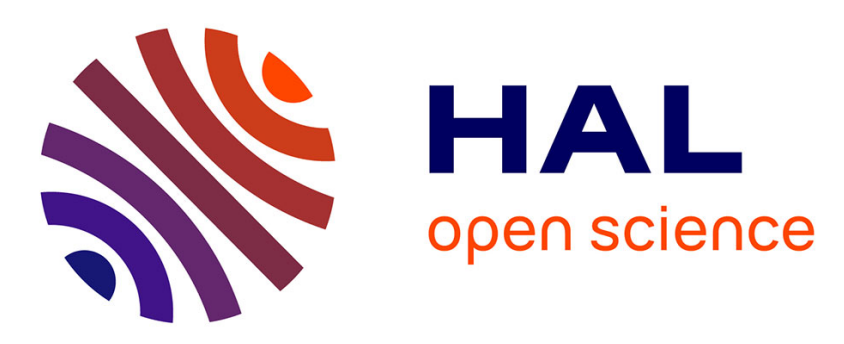

\title{
Impact reduction during running: efficiency of simple acute interventions in recreational runners
}

Marlène Giandolini, Pierrick J. Arnal, Guillaume Y. Millet, Nicolas Peyrot, Pierre Samozino, Blaise Dubois, Jean-Benoît Morin

\section{- To cite this version:}

Marlène Giandolini, Pierrick J. Arnal, Guillaume Y. Millet, Nicolas Peyrot, Pierre Samozino, et al.. Impact reduction during running: efficiency of simple acute interventions in recreational runners. European Journal of Applied Physiology, 2013, 113 (3), pp.599-609. 10.1007/s00421-012-2465-y . hal-01232306

\section{HAL Id: hal-01232306 \\ https://hal.univ-reunion.fr/hal-01232306}

Submitted on 28 Oct 2016

HAL is a multi-disciplinary open access archive for the deposit and dissemination of scientific research documents, whether they are published or not. The documents may come from teaching and research institutions in France or abroad, or from public or private research centers.
L'archive ouverte pluridisciplinaire HAL, est destinée au dépôt et à la diffusion de documents scientifiques de niveau recherche, publiés ou non, émanant des établissements d'enseignement et de recherche français ou étrangers, des laboratoires publics ou privés. 


\title{
Impact reduction during running: efficiency of simple acute interventions in recreational runners
}

\author{
Marlène Giandolini · Pierrick J. Arnal • \\ Guillaume Y. Millet • Nicolas Peyrot • \\ Pierre Samozino $\cdot$ Blaise Dubois $\cdot$ Jean-Benoît Morin
}

\begin{abstract}
Running-related stress fractures have been associated with the overall impact intensity, which has recently been described through the loading rate (LR). Our purpose was to evaluate the effects of four acute interventions with specific focus on LR: wearing racing shoes (RACE), increasing step frequency by $10 \%$ (FREQ), adopting a midfoot strike pattern (MIDFOOT) and combining these three interventions (COMBI). Nine rearfootstrike subjects performed five 5-min trials during which running kinetics, kinematics and spring-mass behavior were measured for ten consecutive steps on an instrumented treadmill. Electromyographic activity of gastrocnemius lateralis, tibialis anterior, biceps femoris and
\end{abstract}

Communicated by Jean-René Lacour.

M. Giandolini · P. J. Arnal · G. Y. Millet · J.-B. Morin

University of Lyon, 42023 Saint-Etienne, France

M. Giandolini · P. J. Arnal · G. Y. Millet · J.-B. Morin

Laboratory of Exercise Physiology (EA4338),

42000 Saint-Etienne, France

N. Peyrot

University of La Réunion, UFR SHE, CURAPS-DIMPS

(EA4075), 97430 Le Tampon, France

P. Samozino

Laboratory of Exercise Physiology (EA4338),

University of Savoie, 73376 Le Bourget-du-Lac, France

B. Dubois

Faculty of Medicine, University of Laval, Québec, Canada

J.-B. Morin $(\bowtie)$

Laboratoire de Physiologie de l'Exercice (EA4338), Médecine

du Sport-Myologie CHU Bellevue, Université de Saint-Etienne,

42055 Saint-Etienne Cedex 2, France

e-mail: jean.benoit.morin@univ-st-etienne.fr vastus lateralis muscles was quantified over different phases of the stride cycle. LR was significantly and similarly reduced in MIDFOOT $\left(37.4 \pm 7.20 \mathrm{BW} \mathrm{s}^{-1},-56.9 \pm\right.$ $50.0 \%)$ and COMBI $\left(36.8 \pm 7.15 \mathrm{BW} \mathrm{s}^{-1},-55.6 \pm\right.$ $29.2 \%)$ conditions compared to NORM $(56.3 \pm 11.5$ $\mathrm{BW} \mathrm{s}^{-1}$, both $\left.P<0.001\right)$. RACE $\left(51.1 \pm 9.81 \mathrm{BW} \mathrm{s}^{-1}\right)$ and FREQ $\left(52.7 \pm 11.0 \mathrm{BW} \mathrm{s}^{-1}\right)$ conditions had no significant effects on LR. Running with a midfoot strike pattern resulted in a significant increase in gastrocnemius lateralis pre-activation $(208 \pm 97.4 \%, P<0.05)$ and in a significant decrease in tibialis anterior EMG activity $(56.2 \pm 15.5 \%$, $P<0.05)$ averaged over the entire stride cycle. The acute attenuation of foot-ground impact seems to be mostly related to the use of a midfoot strike pattern and to a higher preactivation of the gastrocnemius lateralis. Further studies are needed to test these results in prolonged running exercises and in the long term.
Abbreviations
BF $\quad$ Biceps femoris
BW Body weight
$F_{\max } \quad$ Maximal vertical ground reaction force
Fzl Magnitude of impact force peak
GL Gastrocnemius lateralis
$k_{\text {leg }} \quad$ Leg stiffness
$k_{\text {vert }} \quad$ Vertical stiffness
LR Vertical mean loading rate
MFS Midfoot strike
$\mathrm{PSF}_{+10 \%}$ Preferred step frequency increased by $10 \%$
RFS Rearfoot strike
$t_{\mathrm{a}} \quad$ Aerial time
TA Tibialis anterior 
$t_{\mathrm{c}} \quad$ Contact time

$t F z 1 \quad$ Time to impact peak

VGRF Vertical ground reaction force

VL Vastus lateralis

$\Delta L \quad$ Maximal leg spring compression during contact

$\Delta z \quad$ Vertical maximal downward displacement of the center of mass during contact

\section{Introduction}

Running is a popular physical activity, but may also be a source of injuries, and notably bone stress fractures. Twenty percent of all sport-related injuries are stress fractures (Snyder et al. 2006), the most frequent being tibial and metatarsal stress fractures, with tibial fractures representing 33-55\% of all stress fractures (Brukner et al. 1996). At each running step, when the foot strikes the supporting ground, a ground reaction force (GRF) of two to three times body weight is rapidly generated (Cavanagh and Lafortune 1980) inducing shock waves that propagate throughout the locomotor system. These repetitive impact shocks have been reported to be a mechanical factor causing stress fractures (Dickinson et al. 1985). Many factors could influence impact magnitude but to our knowledge no multi-factorial experimental study has investigated several of theses factors yet.

Furthermore, "impact" is a rather vague phenomenon that could be characterized by many mechanical parameters. According to Shorten and Mientjes (2011), impact force is characterized by a force pulse transmitted through the foot in a short duration and thus by high frequency. Among the experimentally measurable mechanical features of impact during the running step, Samozino et al. (2008) showed that time to impact force peak $(t F z l)$ and loading rate (LR) were the most discriminant parameters and the most directly related to the magnitude of the foot-ground impact shock as quantified with skin-mounted accelerometers. These factors were better related to the intensity of the shock than the magnitude of the impact force peak $(F z 1)$ (Samozino et al. 2008). LR, identified as the average time derivative of vertical ground reaction force (VGRF) between the beginning of foot-ground impact and the time to impact force peak, is therefore thought to influence the risk of stress fractures. While LR or $t F z 1$ derived from VRGF measurements are expected to be more sensitive to changes in impact force than magnitude of impact force peak $(F z l)$, they also contain a low frequency feature that represents 'non-impact' components and should therefore be interpreted with caution (Shorten and Mientjes 2011).

That being said, a recent systematic review by Zapdoor and Nikooyan (2011) has reported that VGRF for the first peak and the second peak was similar between the control group and the group suffering from stress fractures, whereas LR significantly differed between the two groups. Consequently, although it is hardly conceivable to experimentally test this hypothesis, the recent literature clearly identified a high LR as a key mechanical feature related to the risk of stress fractures in running, as also suggested by Milner et al. (2006) and Davis et al. (2004) in female runners. Although a higher LR may be a consequence rather than a cause of stress fractures apparition, this raises the question of the possibility and the best way(s) to reduce LR in running in order to prevent stress fractures or other running-related injuries related to impact shock. Haris Phuah et al. (2010) observed tensile loads on the posterior tibia during the stance phase of running characterized by a net sagittal bending moment that is principally negative. Such bending moments could also represent an important mechanical stress for the tibia. However, this study was conducted on 20 healthy subjects with no history of lower limb stress fracture, and thus the link between peak bending moments acting on the tibia and tibial stress fractures remains hypothetical. Finally, it is worth noting that impact forces may also be, in some circumstances, a favorable phenomenon. For instance, Fuchs et al. (2001) and Fuchs and Snow (2002) have observed in children that a high-impact training (jumping with ground reaction forces production of about $8 \mathrm{BW}$ ) generated gains in bone mineral content and bone area at the femoral neck.

For a given running mileage, and at a given step frequency (i.e., for a given total amount of steps performed), the magnitude of impact at each step, and thus the potential risk of tibial stress fractures are influenced by the type of foot strike pattern. It has generally been observed that athletes wearing hard-sole shoes or running barefoot change their pattern towards a forefoot or a midfoot strike (MFS) to potentially reduce the magnitude of forces applied at the heel (Hennig and Milani 1995; Lieberman et al. 2010; Squadrone and Gallozzi 2009). This shift in the foot strike pattern is generally associated with changes in the running mechanical pattern towards lower step length and contact time $\left(t_{\mathrm{c}}\right)$ and greater lower limb stiffness and plantarflexion (De Wit et al. 2000). Increased plantarflexion during barefoot running is the result of a higher preactivation of triceps surae muscles (Divert et al. 2005) and a lower pre-activation of the tibialis anterior (TA) (von Tscharner et al. 2003). Furthermore, this "impact-reduction style" induces a decrease or even a complete removal of the impact peak, and a markedly reduced LR (Dickinson et al. 1985). Lieberman et al. (2010) reported that forefoot striking barefoot runners halve their LR compared to shod RFS runners due to more plantarflexion and ankle compliance. As recently observed by Altman and Davis (2011), LR and step frequency in barefoot condition are not 
significantly different from LR in MFS shod conditions. However, step frequency is higher, for both conditions compared to RFS (Altman and Davis 2011). As a result, the lower foot-ground impact shock associated with the forefoot strike running pattern has very recently been proposed as contributing to a lower risk of running-related injuries in collegiate runners (Daoud et al. 2012). However, even if they highlighted a significant lower LR in barefoot compared to shod conditions, Hamill et al. (2011) observed no significant difference between the different shod conditions studied. Conclusions about impact intensity in different footwear conditions are still debated, notably because of the disparity of the experimental methods used (degree of familiarization of subjects with barefoot running, type of shoes used, distance and duration of the testing trials performed).

Changes in stride frequency are also supposed to influence the risk of stress fractures through their effect on the overall running pattern. The foot-ground impact shock intensity during running (quantified from tibial acceleration) was shown to decrease when subjects ran at a step frequency $20 \%$ higher than their preferred one (Hamill et al. 1995). Further, a simulation study recently showed that the probability of stress fractures increases with running mileage but a $10 \%$ reduction in preferred step length (which corresponds ceteris paribus to a $10 \%$ increase in stride frequency) minimizes this probability, particularly at high mileage (Edwards et al. 2009). When focusing on the associated muscular activity in high mileage ( $>45 \mathrm{~km}$ week ${ }^{-1}$ ) RFS runners, the pre-activation of TA and gastrocnemius medialis decreases compared to low mileage $\left(<45 \mathrm{~km} \mathrm{week}^{-1}\right)$ RFS runners (Baur et al. 2011a, b). Increased step frequency may therefore reduce the impact magnitude by moving the point of impact closer to the midfoot, a pattern naturally adopted by barefoot runners and associated with overall reduced foot-ground impact (Altman and Davis 2011; Lieberman et al. 2010). However, in the modern conditions of physical activity and running environment, pure barefoot running seems difficult to conceive, or at least to be practiced safely by most people.

Finally, footwear has also been shown to alter the running gait pattern. The efficiency of footwear cushioning technologies used to decrease the stress on the skeleton is debated but it has been clearly shown that footwear could influence lower limb mechanics (Lohman et al. 2011). For instance, Hennig and Milani (1995) studied 19 shoe models in RFS marathon runners and concluded that footwear influences peak pressure under rearfoot, midfoot and forefoot and alters foot mechanics. Further, shod running increases dorsiflexion, and decreases knee flexion and ankle motion compared to barefoot running (Bishop et al. 2006; Lieberman et al. 2010). Contact and aerial times $\left(t_{\mathrm{a}}\right)$ and $F z l$ are decreased in barefoot compared to shod running (Divert et al. 2005). Also, the possible role of muscular activity during running, especially in different shod conditions, has been investigated in numerous studies. Nigg (1997) proposed the "muscle tuning" concept which stipulates that muscles would be pre-activated in order to create a damped mechanical system at foot-ground impact. An increase in electromyographic (EMG) signal has been reported when wearing hard-sole shoes, especially during the pre-activation phase (Nigg et al. 2003). However, these changes were not significant, not systematic and highly subject- and muscle-dependent (Nigg and Wakeling 2001). These changes in the running pattern have been interpreted as potentially protecting the body from the shocks generated at heel contact (Nigg et al. 2003). Consequently, as habitually barefoot runners clearly and consistently run with lower LR (Altman and Davis 2011; Lieberman et al. 2010), through the associated MFS or forefoot strike pattern, running with shoes specifically designed to put the runner as close to actual barefoot running as possible is expected to provide similar, yet attenuated effects and may therefore be of interest to potentially reduce the risk of stress fractures. This may explain the recent development of "minimalist shoes", i.e., shoes designed to mimic barefoot running, with less motioncontrol technology, cushioning, stiffness, sole drop and weight than standard running shoes (Jenkins and Cauthon 2011; Lohman et al. 2011).

As detailed above, the literature has proposed several factors potentially influencing LR (footwear, stride frequency, foot strike pattern). Thus, we thought a multi-factorial and cross-sectional experiment was an appropriate approach to evaluate the effect of each of these factors on this mechanical feature of the running impact. The purpose of this study was therefore to determine the acute effects of each of the three main interventions hitherto proposed as effective, i.e., MFS, higher step frequency, minimalist footwear, on running mechanics (and especially foot-ground impact shock intensity) and muscular activity. To our knowledge, the abovementioned ways to reduce LR (foot strike pattern, step frequency and minimalist shoes) have never been experimentally and independently tested during the same protocol and concomitantly with synchronized muscular activity measurements. The latter were performed to allow a better interpretation of the changes in the running mechanical pattern, if observed. We also aimed at testing the effects of a combination of these three interventions, seeking to identify the most efficient solution to reduce running impact shocks (quantified through LR) and by extension potentially prevent musculoskeletal pathologies like tibial stress fractures in running.

\section{Methods}

Subjects

Nine young adults, six males and three females (mean \pm SD: $20.8 \pm 4.7$ years, $\quad 66.2 \pm 10.1 \mathrm{~kg}, \quad 171 \pm 6 \mathrm{~cm}), \quad$ were 
included after giving their informed written consent to participate in this study, which was approved by the local ethical committee and in agreement with the Declaration of Helsinki. All of them were rearfoot strikers (see below), and practiced various sports $\left(10.3 \pm 3.71 \mathrm{~h}\right.$ week $\left.^{-1}\right)$, including recreational running. They were not presenting recent muscular, joint or bone injuries, receiving any medication or wearing orthotics that could interfere with their running pattern according to the participants self-report and the medical examination performed during the familiarization session.

\section{Experimental protocol}

The protocol consisted of two sessions separated by about 2 weeks: a familiarization and inclusion session (session 1), and an experimental session (session 2). During session 1,17 subjects were initially recruited. They warmed up for 5 min on an instrumented treadmill (HEF Tecmachine, Andrézieux-Bouthéon, France) and were asked to individually set their preferred running speed. To do so, they started to run at $2.5 \mathrm{~m} \mathrm{~s}^{-1}$ for $2 \mathrm{~min}$ and after 2 min they were free to increase or decrease their speed to a selfselected pace, with no feedback provided on its value, according to the method recently proposed by Heiderscheit et al. (2011). Then, the subjects performed a 5-min trial beginning at a random speed (still unknown to them) and were asked to adjust again their preferred running speed. For each subject, the average of the two speeds collected was then retained as their preferred running speed for the second session. After a 5-min rest, subjects ran for $5 \mathrm{~min}$ at their preferred running speed in order to measure their preferred step frequency and their foot strike pattern (RFS or not, according to whether or not an impact peak was present on the VGRF signal). The presence of a force impact peak (of magnitude higher than one BW during the first $50 \mathrm{~ms}$ of contact) for at least nine out of ten consecutive steps was the criterion for RFS pattern and inclusion in the study.

At the beginning of session 2, subjects warmed up on the instrumented treadmill during a 5-min trial at their preferred running speed, which allowed us to confirm both their RFS pattern and preferred step frequency. They performed five 5-min trials at their preferred running speed in five different conditions separated by 2 min of rest and a 2-min "reference condition" trial. The latter corresponded to a back-to-normal run (preferred step frequency, preferred running speed, usual running shoes of the subject) allowing to control the stability of the natural pattern and avoid a potential "memory effect" of one experimental condition on the following one (Mundermann et al. 2002). Finally, a reference condition trial was performed at the end of the protocol. A 20-s sampling of data was performed after 1 min during each of these reference condition runs.
Two shoe models were used: one with typical cushioning and motion-control systems (Kalenji Kiprun $2000^{\mathrm{TM}}$, mass: $360 \mathrm{~g}$, midsole hardness: Asker $75 \mathrm{C}$ at the heel and Asker $84 \mathrm{C}$ at the metatarsals) with a heel height of $28.3 \mathrm{~mm}$ and a metatarsal height of $20.2 \mathrm{~mm}(8.1 \mathrm{~mm}$ drop), and a racing shoe (Kalenji Inspid Comp ${ }^{\mathrm{TM}}$, mass: $215 \mathrm{~g}$, midsole hardness: Asker $61 \mathrm{C}$ at the heel and Asker $74 \mathrm{C}$ at the metatarsals) with a heel height of $23.3 \mathrm{~mm}$ and a metatarsal height of $12.5 \mathrm{~mm}$ (10.8 mm drop). Concerning footwear, we investigated the effects of racing shoes, defined in the sport shoe market between standard cushioning-motion-control shoes and minimalist shoes (e.g., Vibram Fivefingers ${ }^{\mathrm{TM}}$, as used in Squadrone and Gallozzi 2009) in order to study a shoe model closer to the currently most usual race running practice. The five experimental conditions tested were-NORM: cushioned and motioncontrol running shoes with the preferred step frequency and freely chosen (i.e., RFS) running pattern, RACE: racing shoes with the preferred step frequency and freely chosen pattern, FREQ: cushioned and motion-control shoes with running frequency $10 \%$ higher than the preferred step frequency $\left(\mathrm{PSF}_{+10 \%}\right)$ and freely chosen pattern, MIDFOOT: cushioned and motion-control shoes with the preferred step frequency and MFS pattern, COMBI: combination of all experimental conditions (i.e., racing shoes, $\mathrm{PSF}_{+10 \%}$, MFS pattern). Subjects ran at their preferred running speed in all conditions, and step frequencies were set by an audio tone. Three 20-s samplings of data were performed at the end $(4.5 \mathrm{~min})$ of each 5 -min trial without informing subjects about the exact moment of sampling and the variables studied (Morin et al. 2009). The three conditions NORM, FREQ and RACE were assigned in a randomized and counterbalanced order among subjects, to limit potential "memory effect" of some conditions, as detailed by Mundermann et al. (2002). Then, MIDFOOT and COMBI conditions were systematically assigned in this order at the end of the running trials series, because the verbal instruction to adopt a MFS could lead subjects to keep memory of this instruction during the subsequent conditions, although required not to. Concerning the MFS instruction in MIDFOOT and COMBI conditions, subjects were asked to "strike the ground with the middle of the foot, below the metatarsal joints". The overall respect of this instruction by each subject was verified visually throughout the trial, and confirmed a posteriori from the absence of impact peak on the VGRF traces for more than nine steps out of ten.

Mechanical variables

The main running kinetics, kinematics and spring-mass model parameters were quantified from VGRF data collected during the 20-s acquisitions performed with a 
sampling rate of $1,000 \mathrm{~Hz}$ on the instrumented treadmill. This treadmill allows measurements of three-dimensional GRF (Belli et al. 2001; Divert et al. 2005), and to determine $F z l$ (force impact peak), $t F z l$ (time to impact peak) and LR (vertical mean loading rate), which was calculated as the mean value of the time-derivate of VGRF signal within the first $50 \mathrm{~ms}$ of the support phase, and expressed in BW s$~^{-1}$ (e.g., De Wit et al. 2000). Last, $t_{\mathrm{c}}$ and $t_{\mathrm{a}}$ were determined from VGRF signal and expressed in seconds. The preferred step frequency $(\mathrm{Hz})$ was calculated from $t_{\mathrm{c}}$ and $t_{\mathrm{a}}$ as step frequency $=\left(t_{\mathrm{c}}+t_{\mathrm{a}}\right)^{-1}$.

A spring-mass model was used to investigate the effect of the different interventions used on the mechanical behavior of the lower limb during running (e.g., Farley and Gonzalez 1996). According to this model, the leg stiffness $\left(k_{\text {leg }}\right.$ in $\mathrm{kN} \mathrm{m}^{-1}$ ) was calculated from $\operatorname{VGRF}(\mathrm{t})$ measurements as $k_{\text {leg }}=F_{\text {max }} / \Delta L$ with $\Delta L$ the maximum leg spring compression $(\mathrm{m})$ calculated from values of initial leg length $L$ (great trochanter to ground distance in a standing position), running velocity $\left(v\right.$ in $\mathrm{m} \mathrm{s}^{-1}$ ), $t_{\mathrm{c}}$ and vertical maximal downward displacement of the center of mass during contact $(\Delta z$ in $\mathrm{m}$ ) obtained by double integration of the center of mass vertical acceleration (Cavagna 1975):

$\Delta L=L-\sqrt{L^{2}-\left(\frac{v t_{\mathrm{c}}}{2}\right)^{2}}+\Delta z$

Finally, the vertical stiffness was calculated as $k_{\mathrm{vert}}=F_{\text {max }} / \Delta z$ and expressed in $\mathrm{kN} \mathrm{m}^{-1}$.

\section{Muscular activity}

EMG activity of the right vastus lateralis (VL), biceps femoris (BF), gastrocnemius lateralis (GL) and tibialis anterior (TA) muscles was recorded using bipolar silver chloride surface electrodes of $30 \mathrm{~mm}$ diameter (Meditrace 100, Tyco healthcare, Mansfield, Canada). The recording electrodes were taped lengthwise on the skin with respect to the underlying muscle fiber arrangement and located according to recommendations by SENIAM (Hermens et al. 2000) with an inter-electrode distance of $30 \mathrm{~mm}$. The reference electrode was attached to the skin facing the patella. Low impedance $(Z<5 \mathrm{k} \Omega)$ at the skin-electrode surface was obtained by abrading the skin with thin sand paper and cleaning with alcohol. EMG data were recorded with PowerLab system (16/30-ML880/P, ADInstruments, Bella Vista, Australia) with a sampling frequency of $2,000 \mathrm{~Hz}$. The EMG signal was amplified with octal bioamplifier (Octal Bioamp, ML138, ADInstruments) with a bandwidth frequency ranging from 5 to $1,000 \mathrm{~Hz}$ (input impedance $=200 \mathrm{M} \Omega$, common mode rejection ratio $=$ $85 \mathrm{~dB}$ ), transmitted to the $\mathrm{PC}$ and analyzed with LabChart 7.3 software (ADInstruments). VGRF and EMG were synchronized on LabChart 7.3. EMG activity of each muscle was quantified using the root mean square (RMS) and recorded during the following phases of the running cycle: (1) pre-contact phase $50 \mathrm{~ms}$ before impact, (2) impact phase during the $30 \mathrm{~ms}$ (average $t F z l$ of the group) following the foot-ground contact as detected by a $30-\mathrm{N}$ threshold, (3) braking phase from impact to midstance, (4) support phase from foot ground contact to toe-off, and (5) stride cycle phase ranging over two consecutive steps (see details in Fig. 1). RMS data for all these phases in the four other experimental conditions were normalized to NORM condition.

\section{Data analysis and statistics}

Descriptive statistics are presented as mean \pm SD for mechanical variables and as percentage of NORM for EMG RMS data. All parameters were averaged for ten successive steps. Normal distribution was checked by the Shapiro-Wilk normality test and variance homogeneity was tested by the Fisher $F$ test. Mechanical data collected in session 2 were compared between the five experimental conditions and between the six reference conditions. EMG data of the four other experimental conditions were compared to NORM. Actual PSF $+10 \%$ measured during FREQ and COMBI were compared to the theoretical $\mathrm{PSF}_{+10 \%}$ by means of a $t$ test in order to check whether subjects respected the $\mathrm{PSF}_{+10 \%}$ set by audio tone in these two conditions. The minimum detectable change (MDC) was calculated for LR between the first and the second reference conditions values according to Kovacs et al. (2008). The importance of the differences found was assessed through the effect size Cohen's $d$ coefficient (Cohen 1998). The interpretation of the effect size was as follows$0.2 \leq d<0.5$ : small difference, $0.5 \leq d<0.8$ : medium difference, $d>0.8$ : large difference. The significant level was set at $P<0.05$.

\section{Results}

Running mechanics

During session 1, preferred running speed was $3.28 \pm$ $0.65 \mathrm{~m} \mathrm{~s}^{-1}$, with a preferred step frequency of $2.87 \pm$ $0.23 \mathrm{~Hz}$, and thus a $\mathrm{PSF}_{+10 \%}$ of $3.15 \pm 0.26 \mathrm{~Hz}$. The average $t_{\mathrm{c}}$ and $t_{\mathrm{a}}$ were $0.241 \pm 0.033$ and $0.110 \pm 0.025 \mathrm{~s}$, respectively. Average values for the group were $1.66 \pm 0.184$ BW for $F z l, 0.033 \pm 0.004 \mathrm{~s}$ for $t F z 1,50.7 \pm 9.11 \mathrm{BW} \mathrm{s}^{-1}$ for LR and $14.5 \pm 1.64 \mathrm{kN} \mathrm{m}^{-1}$ for $k_{\mathrm{leg}}$.

During session 2, normality was obtained for every sample of data $(P<0.05)$, and variance homogeneity was confirmed for eight parameters out of ten $(P<0.05)$. There 
Fig. 1 Synchronized raw EMG and vertical ground reaction force data (VGRF) for a typical subject in MIDFOOT (black lines) and NORM (gray lines) for tibialis anterior (TA) and gastrocnemius lateralis (GL) during the five phases of the running step cycle: pre-contact (1), impact phase (2), braking phase (3), support phase (4) and stride cycle (5)

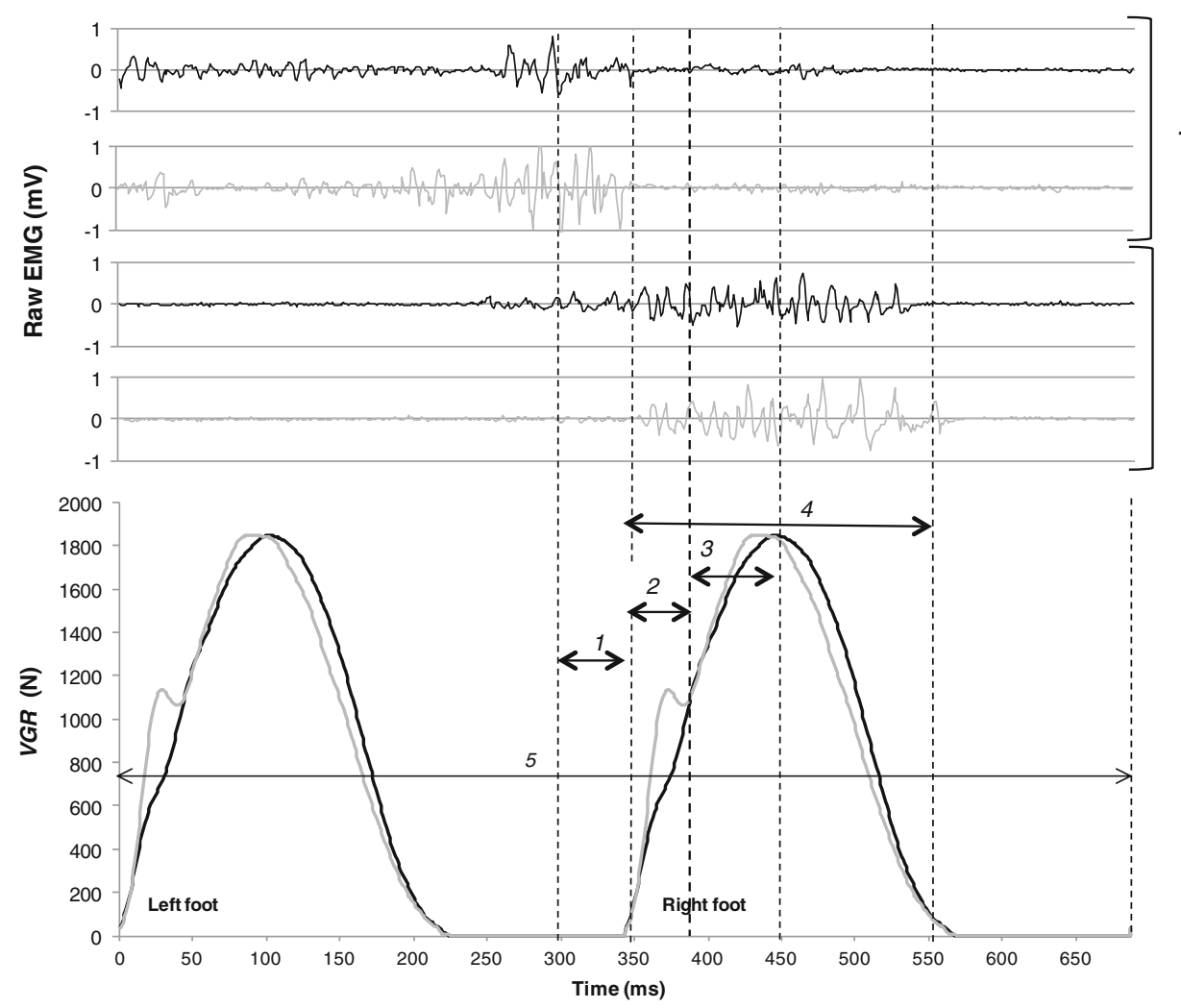

TA was no significant difference $(P=0.61)$ between the actual $\mathrm{PSF}_{+10 \%}$ measured during FREQ $(3.09 \pm 0.20 \mathrm{~Hz})$ and COMBI $(3.10 \pm 0.26 \mathrm{~Hz})$ and the calculated and set $\mathrm{PSF}_{+10 \%}$. There was no significant difference between the six reference conditions indicating that no "memory" or "fatigue" effects was detected, i.e., subjects maintained their natural pattern throughout the protocol in these reference conditions.

Concerning kinetic measurements (Table 1), only MIDFOOT and COMBI resulted in a complete removal of the impact peak. $F z 1$ and $t F z l$ were not different between NORM and RACE $(P=0.877)$ and between NORM and FREQ $(P=0.630)$. MDC for LR was $3.90 \mathrm{BW} \mathrm{s}^{-1}$. Compared to NORM, LR decreased to a similar extent during MIDFOOT $(-56.9 \pm 50 \%, P<0.001$, large effect size of 1.65$)$ and COMBI $(-55.6 \pm 29.2 \%, P<0.001$, large effect size of 1.70) conditions. LR was not altered by RACE or FREQ $(P=0.310$ and 0.305 , small effect size of 0.452 and 0.317 , respectively).

The main running step temporal variables (Table 2) showed no change in $t_{\mathrm{c}}$ among the conditions tested, whereas $t_{\mathrm{a}}$ was lower for FREQ than for NORM $(P<0.04)$ and MIDFOOT $(P<0.02)$. As shown in Table 3 , the spring-mass variable $k_{\text {leg }}$ increased significantly in COMBI compared to NORM $(P<0.02)$. Similarly, $k_{\text {vert }}$ was found to be significantly higher in COMBI and FREQ $(P<0.05)$ than in NORM.
Muscular activity

No difference in muscular activity magnitude was found in FREQ and RACE compared to NORM for all the muscles and EMG variables studied. During the pre-contact phase, GL activity was significantly higher and TA activity significantly lower in MIDFOOT and COMBI compared to NORM (Fig. 2). During the impact phase, no significant difference was found for any condition. No significant correlation was found between muscular activity magnitude during the pre-contact phase and loading rate variables in all conditions. During both braking and support phases, VL activity was significantly lower $(P<0.05)$ in COMBI than in NORM. When considering the entire stride cycle, TA activity was significantly lower $(P<0.05)$ in MIDFOOT and COMBI compared to NORM.

\section{Discussion}

The purpose of this study was to assess the magnitude of the foot-ground impact shock decrease resulting from four simple acute interventions in order to identify the running condition(s) that could potentially reduce the loading rate, and in turn (as supported by recent studies) help prevent tibial stress fractures. The main result of this study is that adopting a midfoot strike running pattern alone 

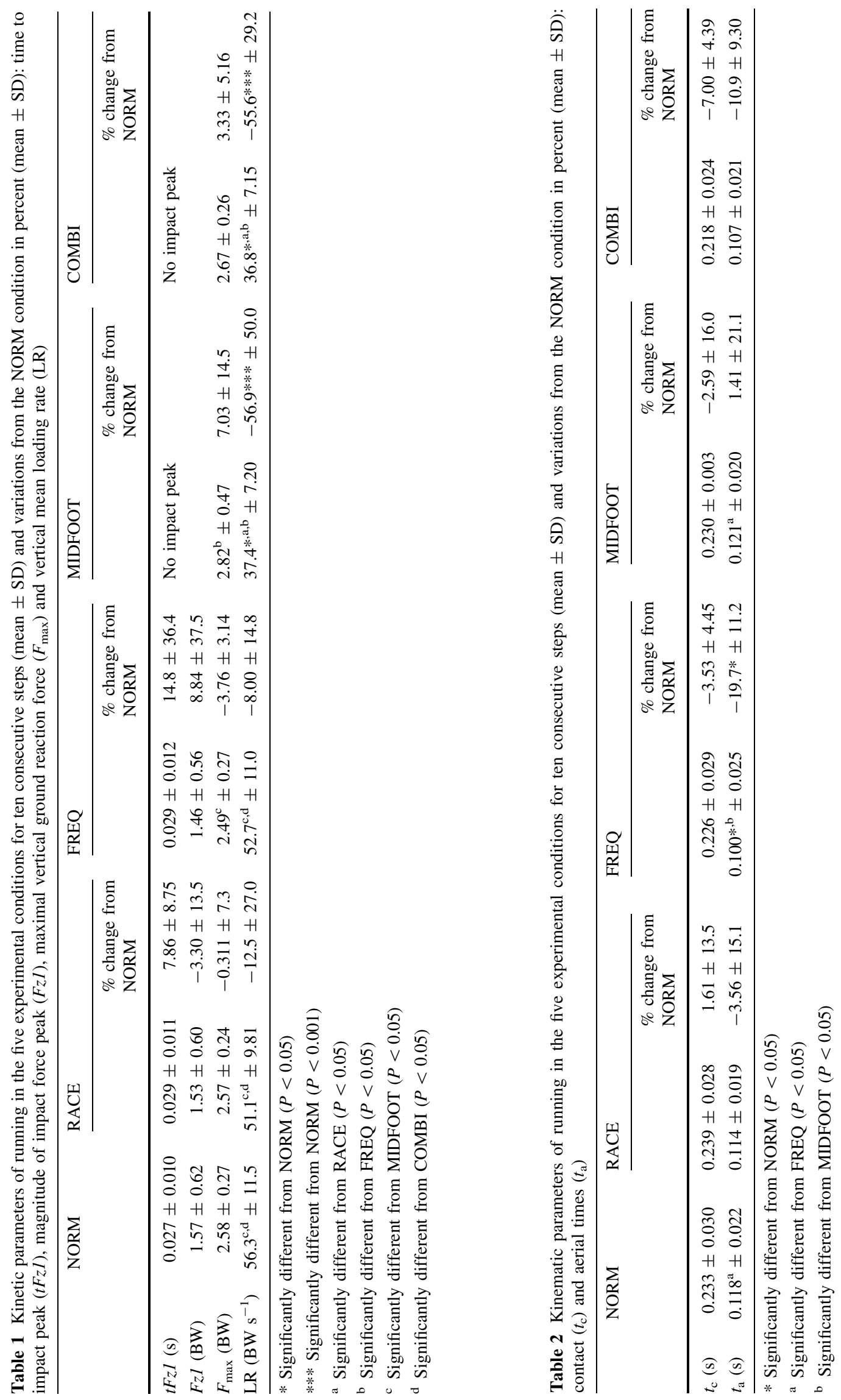


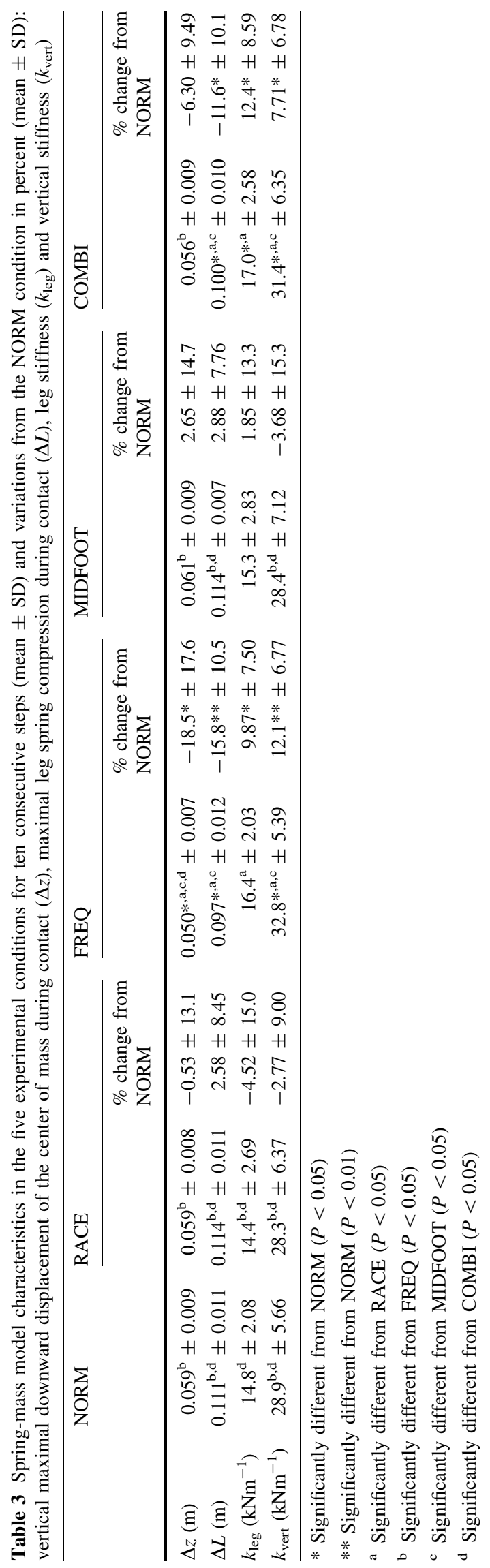

(MIDFOOT) and the combination of the three interventions proposed (COMBI) resulted in a complete removal of the impact peak and an approximately $50 \%$ lower loading rate with non-significant differences between midfoot strike pattern alone and the combination of all solutions. Wearing light racing shoes with no specific cushioning or motioncontrol system, or running with a $10 \%$ higher step frequency did not reduce significantly the loading rate.

The observed effect of an increase in step frequency on running mechanics is in line with that reported by Hamill et al. (1995); in their study, the shock attenuation was significant for a $20 \%$ increase in step frequency, but not for a $10 \%$ increase. Similarly, Heiderscheit et al. (2011) reported that moderate increases in step rate (by 5 and $10 \%$ ) could substantially reduce the overall loading to the hip and knee joints during running. Indeed, in the present study, the attenuation of impact as quantified by the decrease in LR was not significant for a $10 \%$ increase in step frequency. This contradicts the probabilistic stress fracture model of Edwards et al. (2009), which showed an attenuation of the risk of stress fracture probability with a $10 \%$ decrease in stride length. However, these authors considered a theoretical tibial stress occurring during longer distances (from 4.8 to $11.3 \mathrm{~km}$ ) than those considered here. The biomechanical adaptations to an increase in step frequency in order to induce shock attenuation may appear on longer distances. Moreover, the increase in step frequency altered the leg spring behavior; a significant increase in leg stiffness was observed, in accordance with previous findings (e.g., Farley and Gonzalez 1996). In contrary to these spring-mass variables, muscle activity did not change significantly with the increase in step frequency.

The lack of results concerning footwear is probably due to the model of racing shoes used, which had a higher drop $(10.8 \mathrm{~mm})$ than the cushioning-motion-control shoes $(8.1 \mathrm{~mm})$. Despite their lower cushioning under the heel, racing shoes offered a certain protection and thus may have reduced the expected direct incitation for subjects to change their running pattern towards MFS. This is the reason why they were considered and named "racing" shoes rather than minimalist shoes. Thus, our conclusions on footwear were different from the previous findings obtained in barefoot or minimalist shoes conditions, but our study considered racing shoes which (1) are much more used in the current running practice, and (2) have been to date very few considered in experimental protocols about footwear, running mechanics and injuries prevention. Although the model used in our study is not a truly minimalist shoe, the latter have been proposed as an alternative to purely barefoot running because of the difficulty to practice pure barefoot running in the modern environment, especially since (1) most runners are not even used to walk barefoot and (2) pure barefoot running clearly puts the foot 
Fig. 2 Muscle activity expressed in percentage of NORM during the five phases of the running step cycle (see "Methods") in MIDFOOT for: tibialis anterior (TA), gastrocnemius lateralis (GL), biceps femoris (BF) and vastus lateralis (VL). Significant difference for $* P<0.001$

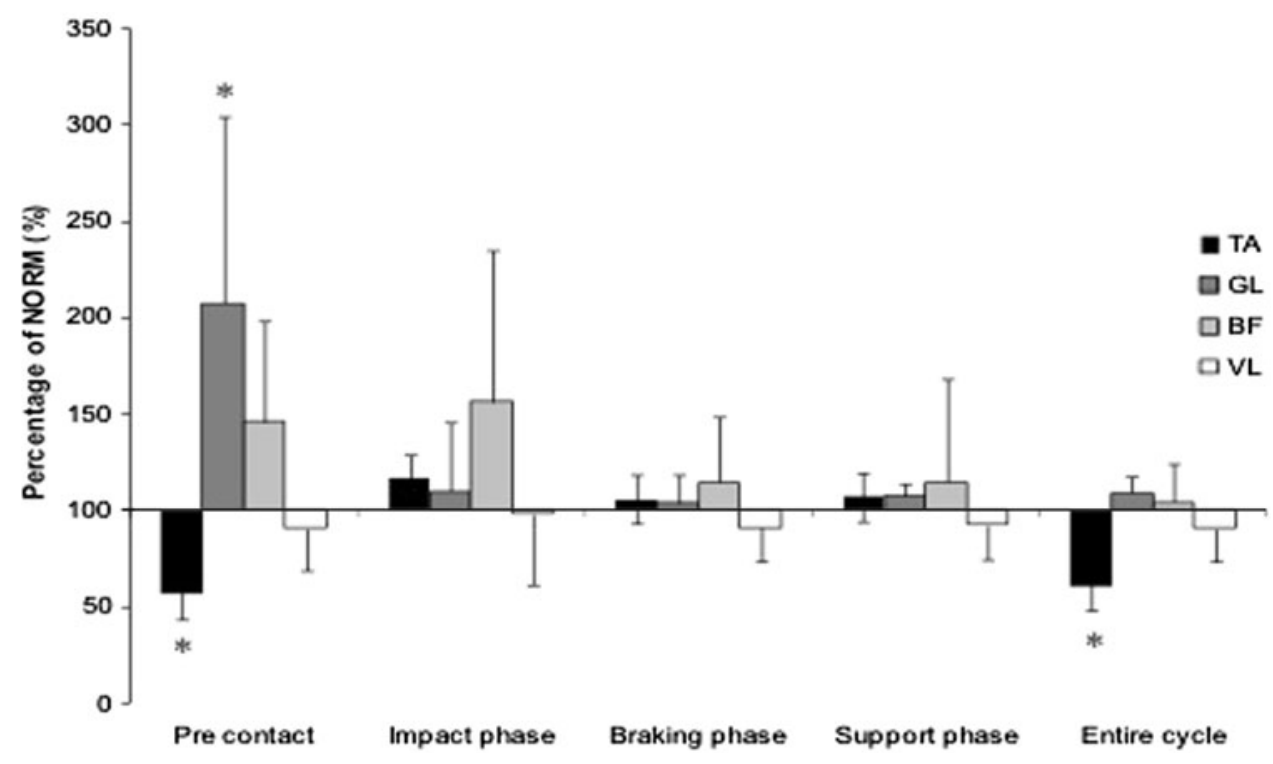

skin structures at risk of lesion (Lohman et al. 2011). Very recent results also pointed that barefoot running does not systematically mean running with a lower metabolic cost, compared to lightweight shoes (Franz et al. 2012). To the best of our knowledge, no study examined the effect of minimalist shoes on EMG activity, but the effect of sole hardness on mechanical and muscular variables has been investigated (Nigg et al. 2003; Nigg and Wakeling 2001). It was found that muscular activity was influenced by sole hardness. However, the group differences in pre-activation of GL were not significant. The authors concluded that changes were subject and muscle-dependent. In the present study, racing shoes did not induce the kinetic, kinematic or muscular variations. However, Lieberman et al. (2010) showed that only $23 \%$ of habitually shod runners (US and Kenyan pooled) shifted from a RFS pattern to a MFS pattern when running barefoot, whereas $82 \%$ of habitually barefoot or minimal runners (wearing Vibram Fivefingers $^{\mathrm{TM}}$ ) adopted a MFS pattern when running barefoot. These authors collected the data at preferred running speed over only $20-25 \mathrm{~m}$, and in our study data were collected during only $5 \mathrm{~min}$ at preferred running speed. It is likely that both these experimental trials were too short to observe adaptations to harder soles, and therefore the adaptation to a harder surface, if existing, may likely occur over longer distances.

The adoption of a MFS pattern reduced LR by about $50 \%$. This was also associated with a complete removal of the impact peak (Fig. 1), which is in accordance with previous findings (Altman and Davis 2011; Daoud et al. 2012; Dickinson et al. 1985; Lieberman et al. 2010). These biomechanical changes were observed for all the natural shod RFS runners included in the present study. The decrease in LR observed here was consistently related to a
MFS pattern. However, adopting a MFS style for longer periods of running and/or in the long-term training process may induce a higher load on the forefoot and midfoot joints than during the acute 5-min conditions tested here. For instance, in barefoot runners adopting a MFS pattern, Squadrone and Gallozzi (2009) showed a decrease in peak pressure under the heel but an increased peak pressure underneath the metatarsal heads. This might lead to a risk of stress injuries located for instance at the metatarsal bones, if the stress applied exceeds the structural and physiological limit of these tissues (Giuliani et al. 2011; Lohman et al. 2011). Queen et al. (2009) have also observed decreases in contact area and maximal force beneath the midfoot in women with history of metatarsal stress fracture compared to a control group, bringing support to gait changes 'post-injury'. However, no biomechanical evaluation 'pre-injury' was performed and thus we cannot conclude whether or not this pattern alteration is directly due to metatarsal stress fractures. A review of literature by Moen et al. (2009) proposes that increasing tension on the tendons of soleus, tibialis posterior and flexor hallucis longus muscles (plantarflexor muscles) induces an increase of the strain on the tibial fascia and in turn to the periosteum. More precisely, these authors showed that a high plantarflexion at the moment of foot strike was a possible risk factor of shin splints. Plantarflexion is increased with a MFS pattern; consequently, we can suppose that the risk of medial tibial stress syndrome (shin splints) is higher too. Concerning muscle activation, adopting the MFS pattern resulted in a higher muscular activity of the GL during the pre-activation phase but not during the support phase (Figs. 1, 2). However, the acute use of this pattern during our study caused delayed-onset muscular pains in the plantarflexors, as reported by most of 


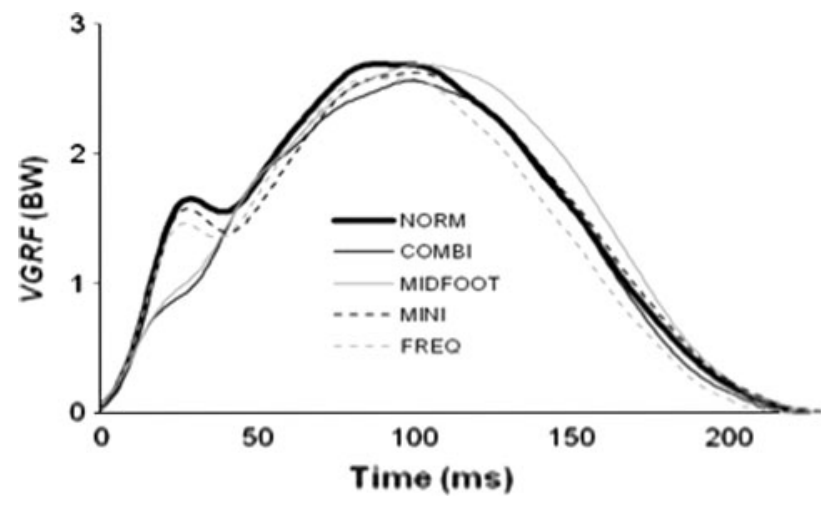

Fig. 3 Mean vertical ground reaction force (VGRF) for the group in the five conditions tested: NORM (black thick full line), COMBI (black thin full line), MIDFOOT (gray thin full line), MINI (black thin dashed line) and FREQ (gray thin dashed line)

the subjects. Moreover, studies investigating Achilles tendinopathy (Baur et al. 2011a, b) observed that in highmileage runners the muscular activity of the GL during the support phase was reduced compared to asymptomatic controls. As previously observed (Nigg and Wakeling 2001), the muscle tuning model supposes that pre-activation increases the stiffness of the muscle-tendon complex, which in turn induces an impact force absorption and a decrease in vibrations, but also a higher load imposed to the Achilles tendon. Nevertheless, in these studies, it was not known whether these changes were a cause or a consequence of the pathology. It is likely that pre-activation of the GL is in fact needed in MFS since the plantarflexors need to counteract the dorsiflexor moment created during the MFS pattern. Recently, Larson et al. (2011) indicated that during a marathon run, up to $93 \%$ of recreational distance runners contact the ground initially on the rearfoot and that the percentage of midfoot strikers decreases with running distance within the marathon. These authors also found no significant relationship between foot strike pattern and race times in marathon runners, in contrary to Hasegawa et al. (2007) who studied elite runners during half-marathon.

Our results show that the most efficient solution for acutely reducing LR is to run with a MFS pattern ( $\sim-57 \%, 95 \% \mathrm{CI}:-58.0$ to 55.9 ) compared to a combination of the three solutions tested $(\sim-56 \%, 95 \%$ CI: -56.2 to 54.9$)$, since it has similar effects, but for a lower quantity of interventions (Fig. 3). Concomitantly with this change in the running mechanical pattern, this led to higher GL activity and lower TA activity during the pre-activation phase. The reduced LR observed in habitual forefoot strikers or resulting from a shift from a rearfoot strike to the MFS pattern in habitual rearfoot strikers as observed in the present study may be associated with a lower risk of running-related injuries, and especially tibial stress fractures
(Daoud et al. 2012). However, it may also cause collateral noxious effects such as metatarsal stress injuries, shin splints, and muscular and tendon injuries if not carefully and progressively conducted (Lohman et al. 2011). Further studies should determine whether the transition towards a consistent MFS pattern in the long-term is possible and not associated with other risks of injuries such as Achilles tendinopathy. Recent data of Crowell and Davis (2011) showed that, after a 2-week gait retraining consisting in visual feed-backs and instructions to "run softer, make their footfalls quieter, and keep the acceleration peaks below the line", a $30 \%$ lower loading rate was still observed 1 month after this protocol. We can thus hypothesize that the "MFS transition" is possible in the long-term with an appropriate and progressive training allowing this transition to be efficient and safe. With reference to the current debate revolving around the benefits/limits of barefoot running (e.g., Jenkins and Cauthon 2011; Krabak et al. 2011), our results show that running "barefoot-like", i.e., with a midfoot strike pattern may be an effective solution to reduce the magnitude of impact, as quantified through the loading rate.

\section{References}

Altman AR, Davis IS (2011) Comparing barefoot running to an altered strike patterns. Med Sci Sports Exerc 43(5):S717

Baur H, Hirschmuller A, Muller S, Cassel M, Mayer F (2011a) Is EMG of the lower leg dependent on weekly running mileage? Int J Sports Med. doi:10.1055/s-0031-1286250

Baur H, Muller S, Hirschmuller A, Cassel M, Weber J, Mayer F (2011b) Comparison in lower leg neuromuscular activity between runners with unilateral mid-portion Achilles tendinopathy and healthy individuals. J Electromyogr Kinesiol 21(3):499-505

Belli A, Bui P, Berger A, Geyssant A, Lacour JR (2001) A treadmill ergometer for three-dimensional ground reaction forces measurement during walking. J Biomech 34(1):105-112

Bishop M, Fiolkowski P, Conrad B, Brunt D, Horodyski M (2006) Athletic footwear, leg stiffness, and running kinematics. J Athl Train 41(4):387-392

Brukner P, Bradshaw C, Khan KM, White S, Crossley K (1996) Stress fractures: a review of 180 cases. Clin J Sport Med 6(2):85-89

Cavagna GA (1975) Force platforms as ergometers. J Appl Physiol 39(1):174-179

Cavanagh PR, Lafortune MA (1980) Ground reaction forces in distance running. J Biomech 13(5):397-406

Cohen J (1998) Statistical power analysis for the behavioural sciences, 2nd edn. Lawrence Erlbaum Associates, Hillsdale (NJ), p 567

Crowell HP, Davis IS (2011) Gait retraining to reduce lower extremity loading in runners. Clin Biomech (Bristol, Avon) 26(1):78-83

Daoud AI, Geissler GJ, Wang F, Saretsky J, Daoud YA, Lieberman DE (2012) Foot strike and injury rates in endurance runners: a retrospective study. Med Sci Sports Exerc. doi: 10.1249/MSS.0b013e3182465115 
Davis I, Milner C, Hamill J (2004) Does increased loading rate lead to tibial stress fractures? a prospective study. Med Sci Sports Exerc 36(S5):S58

De Wit B, De Clercq D, Aerts P (2000) Biomechanical analysis of the stance phase during barefoot and shod running. J Biomech 33(3):269-278

Dickinson JA, Cook SD, Leinhardt TM (1985) The measurement of shock waves following heel strike while running. J Biomech 18(6):415-422

Divert C, Mornieux G, Baur H, Mayer F, Belli A (2005) Mechanical comparison of barefoot and shod running. Int $\mathrm{J}$ Sports Med 26(7):593-598

Edwards WB, Taylor D, Rudolphi TJ, Gillette JC, Derrick TR (2009) Effects of stride length and running mileage on a probabilistic stress fracture model. Med Sci Sports Exerc 41(12):2177-2184

Farley CT, Gonzalez O (1996) Leg stiffness and stride frequency in human running. J Biomech 29(2):181-186

Franz JR, Wierzbinski CM, Kram R (2012) Metabolic cost of running barefoot versus shod: is lighter better? Med Sci Sports Exerc. doi:10.1249/MSS.0b013e3182514a88

Fuchs RK, Snow CM (2002) Gains in hip bone mass from highimpact training are maintained: a randomized controlled trial in children. J Pediatr 141(3):357-362

Fuchs RK, Bauer JJ, Snow CM (2001) Jumping improves hip and lumbar spine bone mass in prepubescent children: a randomized controlled trial. J Bone Miner Res 16(1):148-156

Giuliani J, Masini B, Alitz C, Owens BD (2011) Barefoot-simulating footwear associated with metatarsal stress injury in 2 runners. Orthopedics 34(7):e320-e323

Hamill J, Derrick TR, Holt KG (1995) Shock attenuation and stride frequency during running. Hum Mov Sci 14:45-60

Hamill J, Russell EM, Gruber AH, Miller R (2011) Impact characteristics in shod and barefoot running. Footwear Sci 3(1):33-40

Haris Phuah A, Schache AG, Crossley KM, Wrigley TV, Creaby MW (2010) Sagittal plane bending moments acting on the lower leg during running. Gait Posture 31(2):218-222

Hasegawa H, Yamauchi T, Kraemer WJ (2007) Foot strike patterns of runners at the $15-\mathrm{km}$ point during an elite-level half marathon. J Strength Cond Res 21(3):888-893

Heiderscheit BC, Chumanov ES, Michalski MP, Wille CM, Ryan MB (2011) Effects of step rate manipulation on joint mechanics during running. Med Sci Sports Exerc 43(2):296-302

Hennig EM, Milani TL (1995) In-shoe pressure distribution for running in various types of footwear. $\mathrm{J}$ Appl Biomech 11:299-310

Hermens HJ, Freriks B, Disselhorst-Klug C, Rau G (2000) Development of recommendations for SEMG sensors and sensor placement procedures. J Electromyogr Kinesiol 10(5):361-374

Jenkins DW, Cauthon DJ (2011) Barefoot running claims and controversies: a review of the literature. J Am Podiatr Med Assoc 101(3):231-246

Kovacs FM, Abraira V, Royuela A, Corcoll J, Alegre L, Tomas M, Mir MA, Cano A, Muriel A, Zamora J, Del Real MT, Gestoso M, Mufraggi N (2008) Minimum detectable and minimal clinically important changes for pain in patients with nonspecific neck pain. BMC Musculoskelet Disord 9:43

Krabak BJ, Hoffman MD, Millet GY, Chimes GP (2011) Barefoot running. PM R 3(12):531-535
Larson P, Higgins E, Kaminski J, Decker T, Preble J, Lyons D, McIntyre K, Normile A (2011) Foot strike patterns of recreational and sub-elite runners in a long-distance road race. J Sports Sci. doi:10.1080/02640414.2011.610347

Lieberman DE, Venkadesan M, Werbel WA, Daoud AI, D’Andrea S, Davis IS, Mang'eni RO, Pitsiladis Y (2010) Foot strike patterns and collision forces in habitually barefoot versus shod runners. Nature 463(7280):531-535

Lohman EB III, Balan Sackiriyas KS, Swen RW (2011) A comparison of the spatiotemporal parameters, kinematics, and biomechanics between shod, unshod, and minimally supported running as compared to walking. Phys Ther Sport 12(4):151-163

Milner CE, Ferber R, Pollard CD, Hamill J, Davis IS (2006) Biomechanical factors associated with tibial stress fracture in female runners. Med Sci Sports Exerc 38(2):323-328

Moen MH, Tol JL, Weir A, Steunebrink M, De Winter TC (2009) Medial tibial stress syndrome: a critical review. Sports Med 39(7):523-546

Morin JB, Samozino P, Peyrot N (2009) Running pattern changes depending on the level of subjects' awareness of the measurements performed: a "sampling effect" in human locomotion experiments? Gait Posture 30(4):507-510

Mundermann A, Nigg BM, Stefanyshyn DJ, Humble RN (2002) Development of a reliable method to assess footwear comfort during running. Gait Posture 16(1):38-45

Nigg BM (1997) Impact forces in running. Curr Opin Orthop $8(\mathrm{VI}): 43-47$

Nigg BM, Wakeling JM (2001) Impact forces and muscle tuning: a new paradigm. Exerc Sport Sci Rev 29(1):37-41

Nigg BM, Stefanyshyn D, Cole G, Stergiou P, Miller J (2003) The effect of material characteristics of shoe soles on muscle activation and energy aspects during running. $\mathrm{J}$ Biomech 36(4):569-575

Queen RM, Abbey AN, Chuckpaiwong B, Nunley JA (2009) Plantar loading comparisons between women with a history of second metatarsal stress fractures and normal control. Am J Sports Med 37(2):390-395

Samozino P, Morin J-B, Mermet V, Barla C, Oullion R, Baly L, Belli A (2008) What is the best parameter to quantify shocks during heel-toe running? In: Proceedings of the 13th annual congress of the European college of sport science, Estoril, Portugal, 9-12 July 2008, p 91

Shorten MR, Mientjes MIV (2011) The 'heel impact' force peak during running is neither 'heel' nor 'impact' and does not quantify shoe cushioning effects. Footwear Sci 3(1):41-58

Snyder RA, Koester MC, Dunn WR (2006) Epidemiology of stress fractures. Clin Sports Med 25(1):37-52

Squadrone R, Gallozzi C (2009) Biomechanical and physiological comparison of barefoot and two shod conditions in experienced barefoot runners. J Sports Med Phys Fit 49(1):6-13

von Tscharner V, Goepfert B, Nigg BM (2003) Changes in EMG signals for the muscle tibialis anterior while running barefoot or with shoes resolved by non-linearly scaled wavelets. J Biomech 36(8):1169-1176

Zapdoor AB, Nikooyan AA (2011) The relationship between lowerextremity stress fractures and the ground reaction force: a systematic review. Clin Biomech 26:23-28 